to the unavoidable torture incident to such an occasion, and, at the same time, subjected him to an equal risk of losing with saving his life, the profession and the public would have been satisfied; and, if the event had proved favorable, the act would have been lauded as a splendid affair. As it is, the case has hardly excited any notice or attention. It has been very justly remarked by a distinguished European surgeon, that a surgeon, on commencing an operation, ought to feel chagrined because he was compelled to do like a savage what he had not knowledge sufficient to accomplish like a skilful man.

In the case under consideration the cure was very judiciously attempted by the combination of medication and pressure. By making the pressure on the distal side of the tumor, it is believed, Dr. G. is unprecedented, especially in cases of axillary aneurism.

M. Vernet attempted this method in a case of inguinal aneurism; but the pulsations were so increased, and the inconvenieuce so great, that it had to be abandoned. "This method," remarks Velpeau," has been generally blamed, even by those who have adopted the idea of Brasdor on the subject of ligature; but yet it does not seem worthy of entire rejection. If, for example, it were necessary to treat an aneurism, above which it would be impossible, or at least highly dangerous, to apply compression or ligature; if, on the other hand, no important branch were furnished between the cardiac extremity and the free part of the tumor, it is by no means certain that, by compressing the artery on this latter point, you will not succeed in suspending the circulation in the aneurism, in occasioning the formation of a solid coagulum in its cavity, and, in short, of producing an obliteration of the arterial canal, and a perfect cure of the disease."

That which this eminent and Jearned French surgeon conceived to be barily possible, has been shown, in the present case, to be not only possible, but safe, easy and practicable. And, in fact, this instance, taken in connection with four or five other cases in which cures have recently been accomplished by compression made on the artery between the tumor and the heart, intimate in the strongest manner that the ancient method of cure by pressure has too soon been proscribed. And when it is considered that these cures by compression have occurred in succession in different hospitals, and under the care of different surgeons, we have reason to believe that the early plan of cure by pressure will be revived, improved, and probably, by an adoption, to some extent, of the method of Valsalva, supersede, in many cases, the modes of Anel, Hunter or Brasdor.

\title{
EXTRACTION OF A BROKEN NEEDLE FROM THE HAND.
}

By Estes Howe, M.D., Cambridge, Mass.

[Yom nunicuted for the Boston Medical and Surgical Journal.]

There are few things more embarrassing, in ordinary practice, than being called upon for advice, in a case where a needle has been thrust 
into the flesh, and is supposed to be still present. Very often the evidence of its presence is so equivocal, that one feels very doubtful as to the expediency of an exploration with the scalpel, even at the request of the patient, and still more so as to advising or urging the use of the knife. Yet where a needle is really present, in the hand or foot, or near an articulation, the importance of immediate extraction will not be denied. And where there is no reasonable doubt of the presence of a needle, its exact position, and the direction of its axis, are extremely difficult to determine. We are not at liberty to explore, by incisions in every direction, as we might on the subject, but must content ourselves, at most, with a moderate crucial incision; and it is too often the case that after a search for some minutes, unsuccessfully, our conviction of the existence of the object of our search, " oozes out at the end of our fingers," like Bob Acre's courage-the patient " is sure it's not there," or " it feels better and may work out"-while our backs ache, our eyes are dim with looking, our fingers are tired of poking, and we give it up, in a woful uncertainty as to the case, but quite sure that the patient has an ugly wound to no purpose. To be assured, therefore, of the presence of a needle, and within very small limits, of its exact seat, and the direction of its axis, is no small thing in such cases. Feeling confidence in our diagnosis, we may boldly continue our explorations to complete success-being always able to assure our patient, beyond a doubt, that we shall ultimately succeed.

CASE.-Mrs. F., while washing, thrust into the palmar surface of the right hand, about an inch and a half anterior to the pisiform bone, something sharp-probably a needle. Upon examination of the dress she was washing, the half of a needle was found, and the question was, whether the other half was, or was not, buried in the flesh. On examining the place, a sinall puncture was perceived, from which, I was told, no blood had issued, when I saw the patient an hour after the accident. I probed the puncture with the blunt end of a needle, pretty deeply, but could feel nothing, and upon pressure in various directions could not arrive at any unequivocal evidence of the presence of the broken portion of needle. The patient was very reluctant to submit to the scalpel, and I did not feel sufficiently sure to urge her to submit to it, while I was equally unwilling to have her run the risk of losing the usefulness of her right hand (upon which she and four children depended for bread), by suffering the needle to remain, if it were really there. At this moment, the expedient of Mr. Alfred Smee, described in an article in the "Medical Times," of London, occurred to me, and I resorted to it with perfect satisfaction. His plan is to ascertain the existence and position of the needle, by rendering it a magnet. This may very readily be done, by subjecting it for a certain length of time to the action of a moderately powerful magnet. I procured, from a friend, a pretty powerful magnet-a steel bar about a foot long and half inch square-well charged. This I bound upon the arm, placing one pole directly over the seat of the injury. Two hours after, I removed it, and upon bringing a small magnetic needle, abcut an inch and a half long, into the immediate vicinity of the injured 
part, bad the great gratification of perceiving that it was strongly acted upon, being attracted or repelled as 1 presented one or the other pole. By a few experiments I was able to satisfy myself very nearly of the position of one pole of the magnet ; but the exact direction of the axis I was not able to determine without an experiment that I could not well perform with a needle suspended in the ordinary way, upon a point. I therefore magnetized a common sewing needle, and suspended it by a fine silk thread. Upon bringing the affected part very near it, it was obviously influenced, and upon repeated trials uniformly arranged itself in a particular direction, of course parallel to the axis of the imbedded needle. I had now established the presence of the needle beyond all doubt, and its precise position and direction. A very moderate crucial incision enabled me to reach and extract it, though not without some trouble from the extreme timidity, and intolerance of pain, in the patient. I am sure that I could not have induced her to submit to the operation, unless I had had perfect confidence myself in my diagnosis. Perhaps it may be thought that the magnetic needle would have been attracted by the imbedded needle before it was magnetized, but $I$ ascertained satisfactorily, by experiment, that this was not the case.

It is true, so large a magnet is not always at hand, but a smaller one would bave been effectual, and any person possessing an electro-magnetic apparatus might make one of any size. I have been so much pleased with the result in this case, that I shall never use the knife, where I have any doubts, until I have cleared them up in the manner described.

\section{ON THE TREATMENT OF HYDROCELE BY RETAINED INJECTIONS OF IODINE, AND BY THE SE'TON.}

[Communicated for the Boston Medical and Surgica] Journal.

THE original plan of injecting stimulating solutions into the cavity of the tunica vaginalis superseded all other methods which anticipated a radical cure of hydrocele. This practice, which it is well known consists in a temporary retention of the fluid until the irritation caused by it ends in adhesive inflammation, and then in withdrawing it, has lately been so far modified that a solution of iodine in small quantities bas been substituted for port wine and other astringents, and as an essential feature, is allowed to be retained in the scrotal cavity until removed by absorption. If the statistics which have accumulated in the European journals during a few years past are to be relied upon, iodine must be regarded as possessing specific powers over hydrocele. It is, however, difficult to comprehend that there is no bias or exaggeration in the statements published by the advocates of this exclusive practice, yet among them are many distinguished surgeons of the day whose word is beyond all question.

The practice, or discovery as it is claimed to be, of treating hydrocele by retained ioduretted injections, was first adopted by James Ranald Martin, a medical gentleman in the service of the East India Company, ip Bengal, in 1832. Hydrocele is a disease of great frequency in the Esst 